\title{
Characterization of circulating tumor cells in newly diagnosed breast cancer
}

\author{
LU XU $^{1 *}$, SONGLIN JIA ${ }^{1 *}$, HENGYU LI ${ }^{1}$, YUE YU ${ }^{1}$, GUOPING LIU $^{1}$, YANMEI WU ${ }^{1}$, XISHUI LIU ${ }^{1}$, \\ CHAOQIAN LIU ${ }^{1}$, YUE ZHOU ${ }^{2}$, ZHENZHEN ZHANG ${ }^{1,2}$ and YUAN SHENG ${ }^{1}$ \\ ${ }^{1}$ Department of Breast Surgery, Changhai Hospital, The Second Military Medical University, Shanghai 200433; \\ ${ }^{2}$ Biotecan Medical Diagnostics Co., Ltd., Zhangjiang Center for Translational Medicine, Shanghai 201204, P.R. China
}

Received December 25, 2015; Accepted June 2, 2017

DOI: $10.3892 / \mathrm{ol} .2017 .7540$

\begin{abstract}
Identification of circulating tumor cells (CTCs) by surface marker expression and ploidy analysis [immunostaining-fluorescence in situ hybridization (iFISH)] has been shown to be a highly sensitive method in the identification of certain solid cancers. In the present study, iFISH analysis was performed to identify CTCs in 184 patients with newly diagnosed non-metastatic breast cancer, and the distribution of CTC subtypes was characterized based on cytokeratin (CK) expression and ploidy status. It was revealed that CTCs of non-metastatic, aneuploid breast cancers, independent of CK expression profile, can be detected with high sensitivity $(90.76 \%)$ by the iFISH system. Higher CTC counts and sensitivity were observed in patients with increased tumor size burden and unfavorable hormone receptor status. Investigation of CTC subtypes based on ploidy analysis indicated that triploid CTCs constituted the majority of CTCs evaluated. While CK-positive CTCs were detected in a small cohort of patients, an overall low rate of $\mathrm{CK}$ expression was observed in the 18 patient samples evaluated. Of note, $\mathrm{CK}$ expression was rare in CTCs detected in patients with Herceptin 2 (Her2)-positive or triple-negative [estrogen receptor (ER)-, progesterone receptor (PR)- and Her2-negative], indicating that lack of ER and PR may result in promotion of epithelial-mesenchymal transition and enhancement of tumor aggression.
\end{abstract}

Correspondence to: Professor Yuan Sheng or Dr Zhenzhen Zhang, Department of Breast Surgery, Changhai Hospital, The Second Military Medical University, 168 Changhai Road, Shanghai 200433, P.R. China

E-mail: sheng528yuan@163.com

E-mail: zhenzhen4913@163.com

\section{*Contributed equally}

Key words: circulating tumor cell, breast cancer, ploidy analysis, cytokeratin, tumor size, hormone receptor status

\section{Introduction}

Breast cancer is the most prevalent malignant cancer in women in China and worldwide (1). Outcomes are markedly improved by early breast cancer detection $(2,3)$. Breast cancer screening has been employed to detect lesions at early stages, prior to metastasis. Circulating tumor cells (CTCs) remain a promising avenue for early breast cancer detection as it bypasses the need for ionizing radiation (mammography) or invasive biopsy (4).

Circulating tumor cells (CTCs) are cancer cells that detach from primary or metastatic solid tumors into the vasculature, where they can be sampled from the circulating bloodstream (5). Guidelines for the quantification of CTCs as a breast cancer biomarker have been outlined by the American Society of Clinical Oncology (6). In metastatic breast cancer, the Food and Drug Administration approved the CELLSEARCH ${ }^{\circledR}$ system for routine clinical use in guiding clinical management $(7)$. However, few studies $(4,8)$ have been published in CTC detection for breast cancer prior to metastasis. Mammaglobin-positive CTC detection has previously been performed in women suspected of breast cancer; however, it failed to detect intraductal cancer and $50 \%$ of in situ cancers (4). CTCs also be detected in newly diagnosed inflammatory breast cancer using CELLSEARCH system, which result in a proportion of $54.5 \%$ non-metastatic patients with $>1$ CTCs (8).

The CELLSEARCH ${ }^{\circledR}$ System detects CTCs by detecting the expression of epithelial cell adhesion molecules (EpCAMs) on the tumor cell surface in combination with cytokeratins (CKs) (9). However, CTCs may variably lose these epithelial cell markers during epithelial-mesenchymal transition (EMT) $(3,5,10)$, which can result in low sensitivity and false negatives (11). To overcome these limitations, additional detection methods for CTCs have been proposed including manual assays such as the CytoTrack system (12), magnetophoretic techniques (13) and microfluidic chips such as CTC-iChip ${ }^{\mathrm{TM}}$ (2).

In the present study, a strategy of EpCAM-independent enrichment (14) integrated with immunostaining-fluorescence in situ hybridization (iFISH), which was previously validated in gastric cancer, pancreatic cancer and gliomas $(3,12,13)$, was applied to detect CTCs in patients with newly diagnosed breast cancer. The CTCs were subtyped based on CK expression and 
ploidy analysis, and were associated with tumor size, hormone receptor status and a number of common tumor markers, including carcinoembryonic antigen (CEA) and cancer antigen (CA)15-3.

\section{Patients and methods}

Patients. A total of 184 female patients (age range, between 29 and 87 years) with newly diagnosed breast cancer, 26 female patients with benign breast tumors and 10 female healthy donors were enrolled at Changhai Hospital (Shanghai, China) between February 2014 and June 2015. Peripheral blood samples of the enrolled patients with no prior treatment for breast cancer and healthy donors were collected and evaluated in the present study.

Written consent was provided by all patients. The present study was approved by the Ethics Committee of Changhai Hospital and was performed according to the Declaration of Helsinki principles.

CTC detection. Studies were performed as previously published and according to the manufacturer's protocol of the Cytelligen CTC enrichment kit (cat. no. SEH-003; Cytelligen, Inc., San Diego, CA, USA) (5,15). Briefly, patient blood samples were collected into 7.5-ml tubes containing acid-citrate-dextrose anti-coagulant (BD Biosciences, Franklin Lakes, NJ, USA), followed by thorough mixing by hand and addition of $3 \mathrm{ml}$ of hCTC separation matrix (Cytelligen CTC enrichment kit). The solution was centrifuged at $450 \mathrm{x}$ g for $5 \mathrm{~min}$ at room temperature. Supernatants were collected and incubated with immunomagnetic particles conjugated to anti-leukocytes monoclonal antibodies (Cytelligen CTC enrichment kit), including anti-cluster of differentiation (CD)45, at room temperature for $10 \mathrm{~min}$ with gentle agitation. The solution was subsequently subjected to magnetic separation using a magnetic stand (Promega Corporation, Madison, WI, USA) to remove leukocytes. The magnetic particle-free solution was centrifuged at $500 \mathrm{x}$ g for $2 \mathrm{~min}$ at room temperature. Cell pellets were thoroughly resuspended in cell fixative (Cytelligen CTC enrichment kit) and immediately applied to the coated CTC slides at room temperature for subsequent iFISH analysis. For CK-iFISH, samples were immunostained with a cocktail of Alexa Fluor 594-conjugated monoclonal anti-CD45 and Alexa Fluor 488-conjugated anti-PanCK (CK4, 5, 6, 8, 10, 13 and 18) or anti-Her2 antibodies, all supplied in the Human Tumor Cell Identification kit (cat. no. FSH-002; Cytelligen, Inc.) for $1 \mathrm{~h}$ in the dark at $37^{\circ} \mathrm{C}$. Subsequently, FISH was performed with CEP 8 SpectrumOrange (Vysis; Abbott Laboratories, Abbott Park, IL, USA) using a S500 StatSpin ThermoBrite Slide Hybridization/Denaturation system (Abbott Laboratories), according to the manufacturer's protocol, with the program of denaturing at $73^{\circ} \mathrm{C}$ for $10 \mathrm{~min}$ and hybridizing at $37^{\circ} \mathrm{C}$ for $4 \mathrm{~h}$. CTCs were identified as $\mathrm{DAPI}^{+} / \mathrm{CD}^{4} 5^{-} / \mathrm{PanCK}^{+}$or $\mathrm{Her} 2^{+}$with aneuploid chromosome 8 .

Statistical analysis. Statistical analysis was performed with SPSS 19.0 software (IBM SPSS, Armonk, NY, USA). Differences in CTC number between patients with cancer, patients with benign tumors and healthy donors were compared by Mann-Whitney U test. Positive rates of CTC with or without CK expression were compared using Fisher's exact test. Graphical plots were generated using OriginPro 8 SR0 version 8.0 (OriginLab, Northampton, MA, USA). All the $\mathrm{P}$-values were two-sided. $\mathrm{P}<0.05$ was considered to indicate a statistically significant difference.

\section{Results}

CTCs in breast cancer. A total of 184 patients with newly diagnosed breast cancer, 26 patients with benign breast tumors and 10 healthy individuals were recruited at Changhai Hospital between February 2014 and June 2015. Aneuploid CTCs were detected in 167 of 184 (90.76\%) patients with breast cancer (Table I), with the number of CTCs detected ranging between 0 and 19 cells $/ 7.5 \mathrm{ml}$ blood (median, 2 cells $/ 7.5 \mathrm{ml}$; Table II). The total number of CTCs detected was 675 cells, in which 387 cells (375 cells were CK negative and 12 cells were $\mathrm{CK}$ negative) were identified with triploidy (Fig. 1A). Among the CTCs, CK positivity was detected in only $33(4.88 \%)$ cells from 18 patients (Fig. 1A), and CK-positive CTCs were predominantly detected in luminal A and luminal B tumors (Fig. 1B). Aneuploid CTCs were detected in $8 / 26$ benign tumors $(30.77 \%$ ), which was significantly less compared with that of patients with breast cancer $(\mathrm{P}=0.007)$. Furthermore, all CTC counts in benign tumors were $\leq 3$ cells $/ 7.5 \mathrm{ml}$. No CTCs were identified in healthy donor blood samples (Table I).

Analysis was performed by examining the association between breast cancer CTC counts and common tumor markers, including CEA, CA15-3, CA125, Ki-67, topoisomerase II and p53. Overall, no significant associations were observed between CTC counts and the tumor markers surveyed (data not shown). The relevance of lymph node status, clinical stage and CTC of patients with breast cancer was also analyzed. Generally, no significant associations were observed between CTC count and clinical stage (Fig. 2A) or lymph node status (Fig. 2B).

CTC and tumor size. Of the 184 breast cancer patient samples examined in total, 158 had tumor size data available. Among this cohort, 80 were $\leq 2 \mathrm{~cm}$ (T1), 68 were between $>2$ and $\leq 5 \mathrm{~cm}$ (T2) and 10 were $>5 \mathrm{~cm}$ (T3). Despite the small sample size of T3 patients, the highest CTC counts were detected in this group (range, $0-18$ cells $/ 7.5 \mathrm{ml}$ blood; median, 5.5 cells $/ 7.5 \mathrm{ml}$; Fig. 2C). Triploid CTC counts were notably different between $\mathrm{T} 3$ and T1/T2 patients, with a median number of 4 cells/7.5 ml blood in T3 patients and a median number of 1.5 cells $/ 7.5 \mathrm{ml}$ blood and 1 cells $/ 7.5 \mathrm{ml}$ blood in $\mathrm{T} 1$ and $\mathrm{T} 2$ patients, respectively $(\mathrm{P}=0.048$ and $\mathrm{P}=0.006$; Table II). CTC subtypes based on $\mathrm{CK}$ expression were not significantly different among $\mathrm{T} 1$, T2 and T3 patients (Table II).

CTC and molecular subtyping of breast cancer. Of the 184 patients with breast cancer, 165 had hormone receptor status data available, of which 30 were luminal A $\left(\mathrm{ER}^{+} / \mathrm{PR}^{+}\right.$, Her2 ${ }^{-}$and $\left.\mathrm{Ki} 67<14 \%\right), 89$ were luminal $\mathrm{B}\left(\mathrm{ER}^{+} / \mathrm{PR}^{+}\right.$, Her2 ${ }^{-}$and $\mathrm{Ki} 67 \geq 14 \%$; or $\mathrm{ER}^{+} / \mathrm{PR}^{+}$and Her2 ${ }^{+}$), 35 were Her2-positive $\left(\mathrm{ER}^{-}, \mathrm{PR}^{-}\right.$and $\mathrm{Her}^{+}$) and 12 were triple-negative (ER ${ }^{-} \mathrm{PR}^{-}$and Her2 $)$. CTCs were detected in 24 of the 30 luminal A patients (80.0\%; range, $0-18$ cells/7.5 ml; median, 2 cells/7.5 ml), 


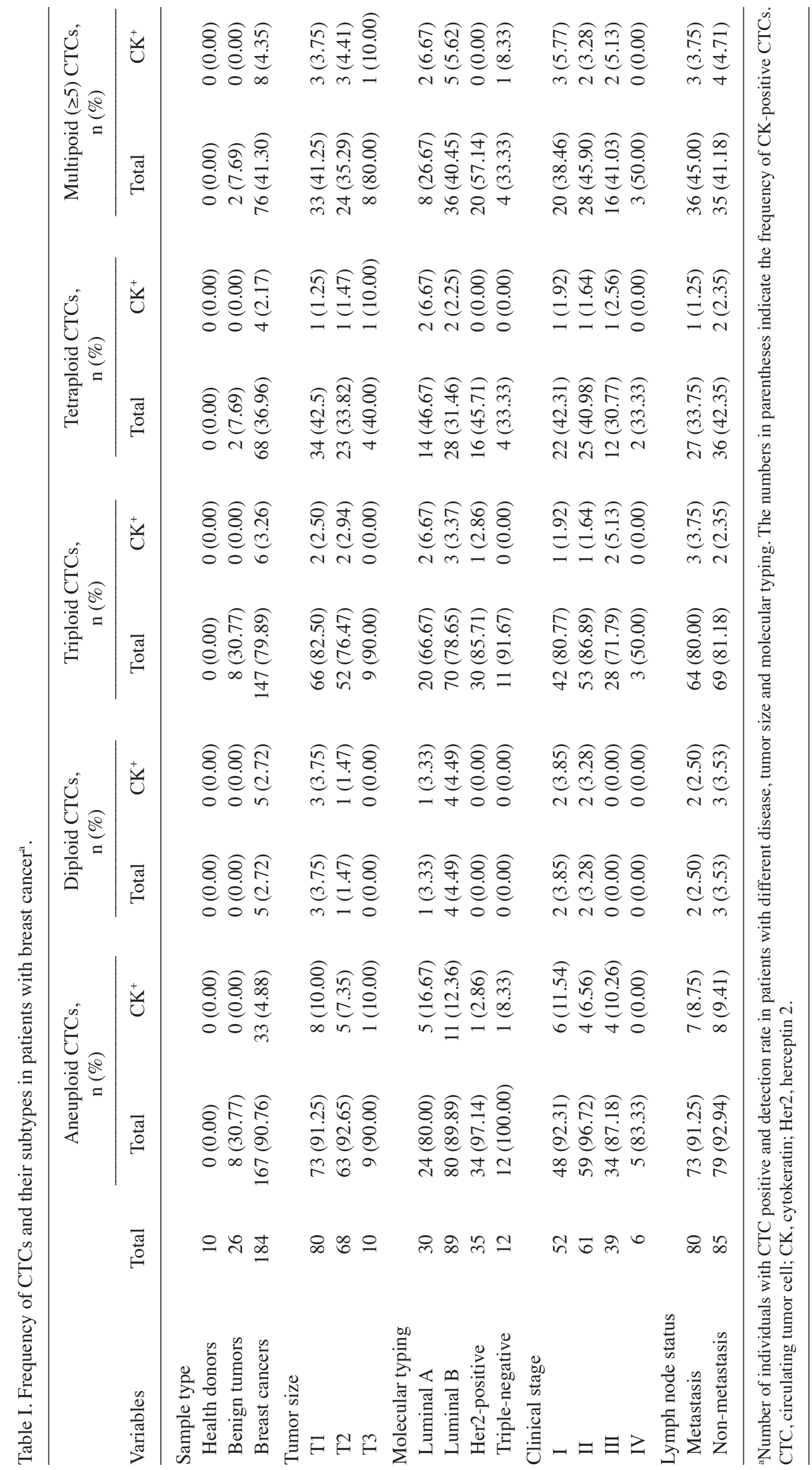




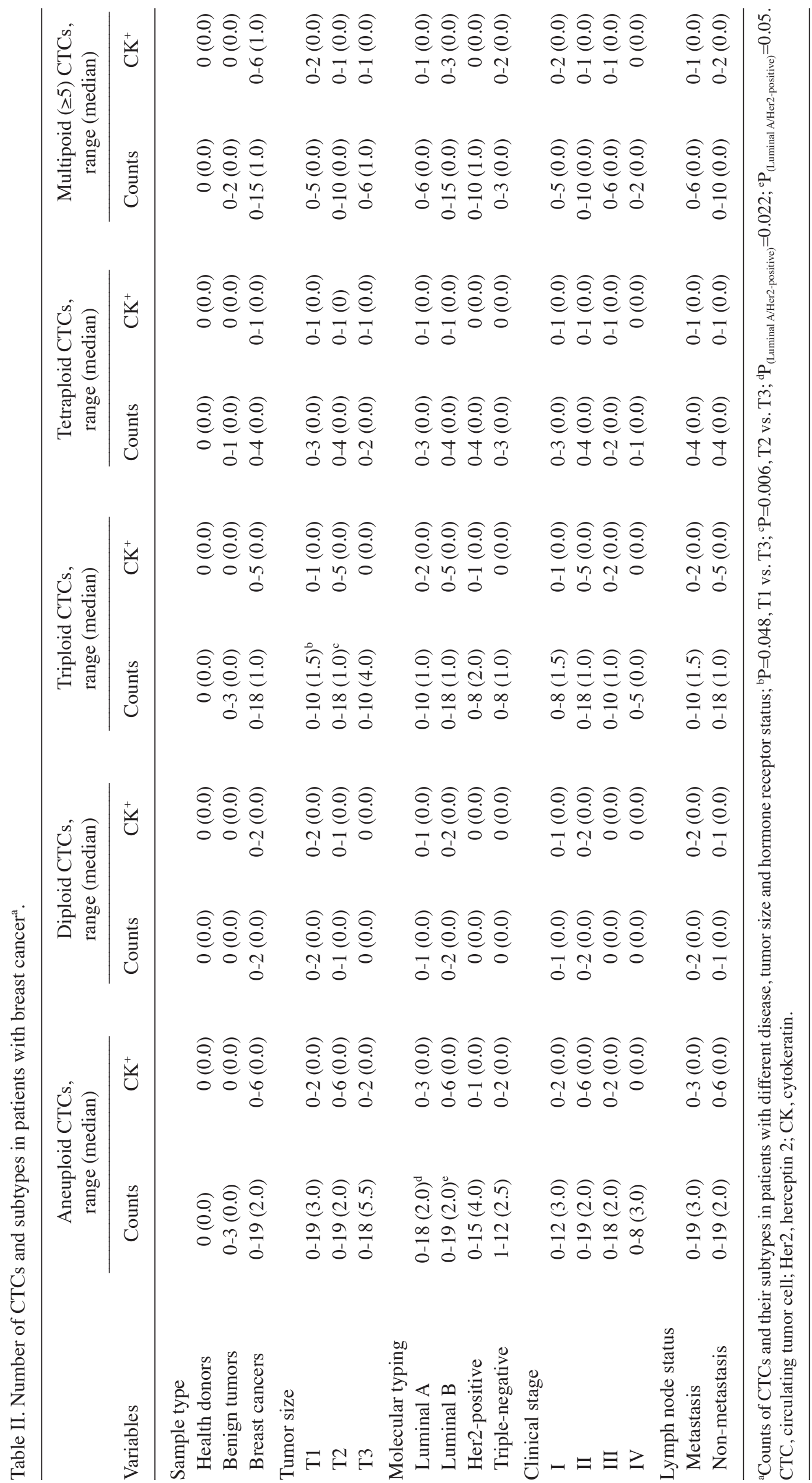


A

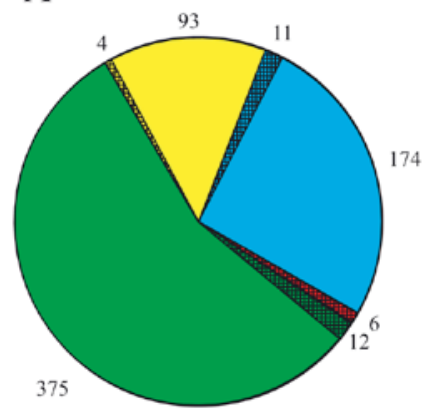

B

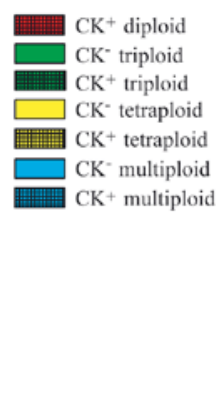

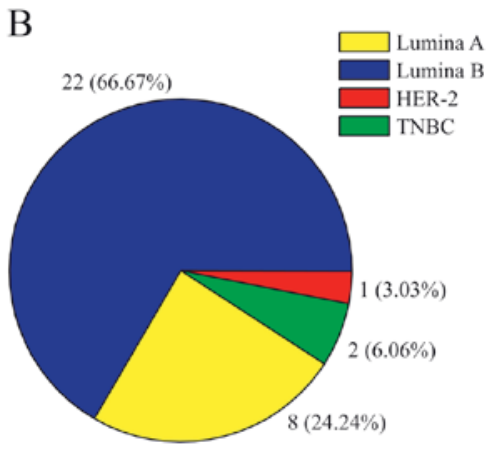

Figure 1. CTC and its subtypes in newly diagnosed patients with breast cancer. (A) Numbers of CTCs with CK-positive or -negative expression, and diploid, triploid, tetraploid and multiploid status are shown. (B) Numbers of CK-positive CTCs according to hormone receptor status are shown. CTC, circulating tumor cell; CK, cytokeratin; Her2, herceptin 2; TNBC, triple negative breast cancer.
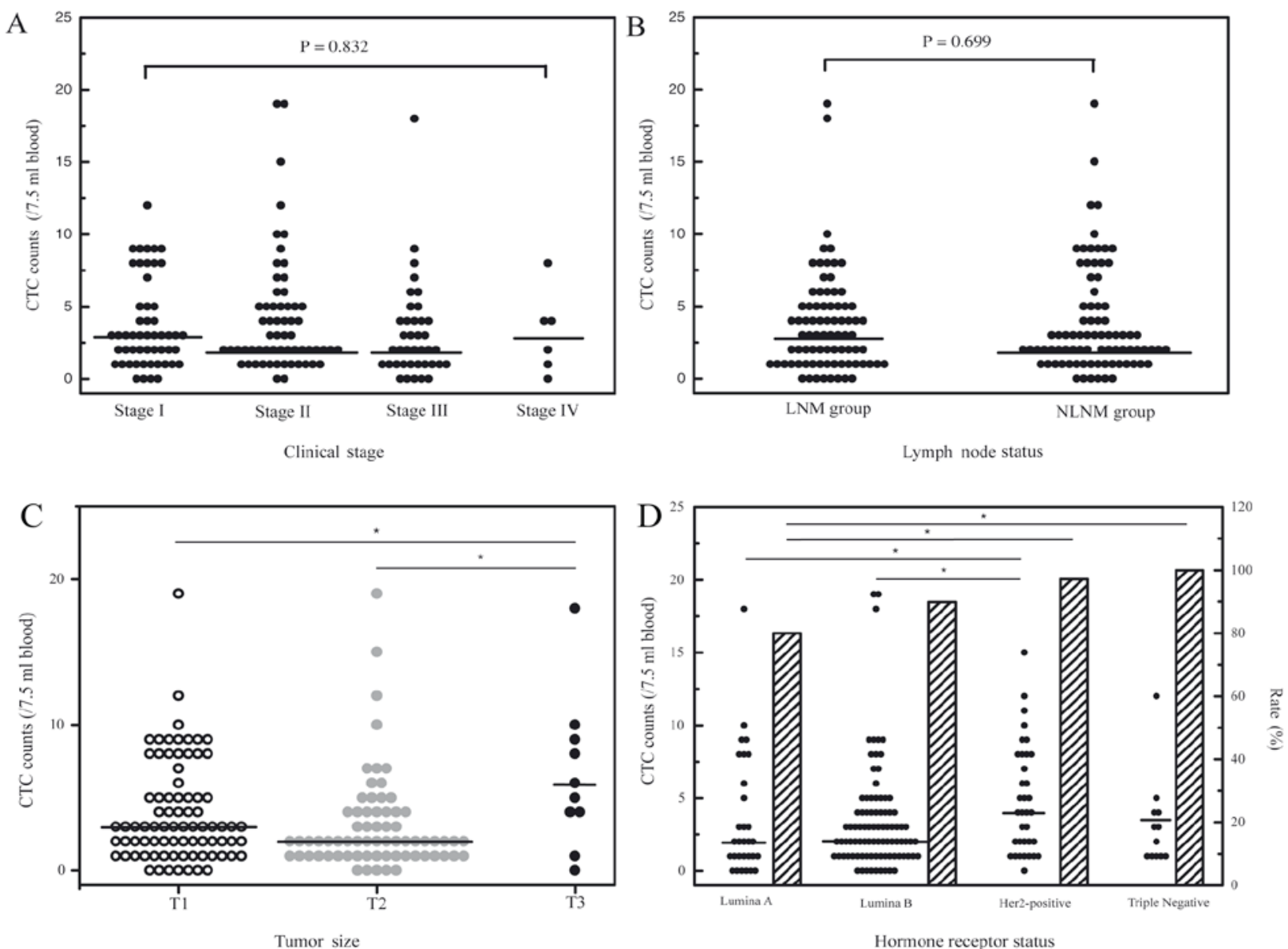

Figure 2. CTC counts and rates in patients with breast cancer. (A) Comparison of CTC detection among patients with different clinical stages. (B) Comparison of CTC detection between patients with LNM and NLNM. (C) CTC counts in patients with different tumor sizes are shown. "P<0.05. (D) CTC counts and detection rates in patients with different hormone receptor status are shown. "P $<0.05$. CTC, circulating tumor cell; LNM, lymph node metastasis; NLNM, no lymph node metastasis; Her2, herceptin 2.

80 of 89 luminal B patients (89.9\%; range, $0-19$ cells $/ 7.5 \mathrm{ml}$; median, 2 cells/7.5 ml), 34 of 35 Her2-positive patients (97.1\%; range, $0-15$ cells $/ 7.5 \mathrm{ml}$; median, 4 cells $/ 7.5 \mathrm{ml}$ ) and 12 of 12 triple-negative patients (100\%; range, $1-12$ cells $/ 7.5 \mathrm{ml}$; median, 2.5 cells $/ 7.5 \mathrm{ml}$ ). Patients with Her2-positive or triple-negative breast cancers had the highest frequency and total number of CTCs compared with those in luminal A or luminal B patients (Fig. 2D; Tables I and II).
CTC subtypes based on karyotype, including triploid, tetraploid and multiploid ( $\geq 5$ ), exhibited a similar distribution in patients with breast cancer with differing hormone receptor status (Table I). However, CK positivity was detected in $22(22 / 363,6.06 \%)$ cells from patients with luminal A or luminal B tumors, which was significantly less compared with that from patients with Her2-positive or triple-negative breast cancers (3/199, 1.51\%; P=0.017; Table III). 
Table III. Different CK expression rate in breast cancer subtypes.

\begin{tabular}{lccc}
\hline $\begin{array}{l}\text { Molecular } \\
\text { typing }\end{array}$ & $\begin{array}{c}\text { Total CTC } \\
\text { counts }\end{array}$ & $\begin{array}{c}\mathrm{CK}^{+} \mathrm{CTC} \\
\text { counts }\end{array}$ & $\begin{array}{c}\text { Expression } \\
\text { rate, } \\
\text { frequency (\%) }\end{array}$ \\
\hline Luminal A & 144 & 6 & $22 / 363(6.06)$ \\
Luminal B & 219 & 16 & \\
Her2-positive & 166 & 1 & $3 / 199(1.51)$ \\
Triple-negative & 33 & 2 & \\
\hline
\end{tabular}

CTC, circulating tumor cell; Her2, herceptin 2; CK, cytokeratin.

\section{Discussion}

In the present study, CTCs were identified in patients with newly diagnosed breast cancer and were revealed to be associated with aneuploidy status by the iFISH system, in addition to tumor surface markers. Although the breast cancers examined were not metastatic with the likely possibility of a low and difficult to detect frequency of CTCs, the present study revealed a relatively higher sensitivity (90.76\%) in CTC detection of breast cancer compare with the sensitivity of $54.5 \%$ in a previously study (8), in which CTC were detected using CELLSEARCH ${ }^{\circledR}$ system. The high sensitivity of CTC detection in breast cancer were identical to that of other solid tumors evaluated with the iFISH system, including gastric (90.5\%) (15), lung (92\%) and esophageal (87\%) cancer (5). In a study of CTCs detected in pancreatic cancer by the same system, 0-2 cells $/ 3.75 \mathrm{ml}$ were detected in benign pancreatic tumors and healthy controls (16). Similarly, 0-3 cells $/ 7.5 \mathrm{ml}$ were detected in benign breast tumors in the present study.

In the present study, the highest rate $(9 / 10,90 \%)$ and CTC counts $(0-18$ cells $/ 7.5 \mathrm{ml}$, median, 5.5 cells $/ 7.5 \mathrm{ml})$ were observed in patients with the greatest tumor size (T3), indicating that tumor size is associated with CTC production. Primary tumor size is a credible predictor of breast cancer metastasis $(7,9,17)$, as corroborated by the findings of the present study. Patients with Her2-positive or triple-negative tumors were also revealed to have more CTCs in rate and counts compared with those in luminal A or luminal B patients. Her2-positive or triple-negative breast cancers carry a worse prognosis with an increased risk of metastasis, compared with luminal A or luminal B patients (18-21). The results of the present study indicated that patients with highly aggressive disease have increased CTCs.

A large proportion (57.25\% of CTCs detected, and $88 \%$ of patients involved) of aneuploid CTCs detected were of triploid subtype. In previous studies, triploid CTCs were detected frequently in gastric (61.2\% of patients) (15), lung (41.9\% of CTCs) and esophageal cancer (37.6\% of CTCs) (17). It was hypothesized that the ratio of triploid CTCs in patients prior to treatment may reciprocally correlate with the chemotherapeutic efficacy (15). In the present study, the most significant difference between $\mathrm{T} 3$ and $\mathrm{T} 1 / \mathrm{T} 2$ patients was the frequency of triploid CTCs, indicating that triploid CTCs may serve a role in tumor progression and treatment.
Among the 167 CTC-positive patients in the present study, CK-positive CTCs were detected in a small cohort of individuals $(18 / 167,10.78 \%)$. CK expression on the cell surface of primary tumors may degrade or become absent in CTCs due to EMT $(3,5,10)$. In the present study, the iFISH system could identify ploidy status in CK-negative aneuploid CTCs in contrast to immunostaining alone. A small rate of patients with CK-positive CTCs were detected in a study evaluating pancreatic (4/22) (16), lung (8/24) and esophageal (4/13) cancer (5). These results suggested that CTC identification by staining of $\mathrm{CKs}$ alone may result in a relatively low frequency and CTC counts.

CTCs with CK positivity were mainly detected in patients with luminal A or luminal B tumors, indicating that almost all CTCs with epithelial features were detected in patients with ER/PR-positive tumors. Epithelial features were absent in CTCs detected in Her2-positive patients, presumably due to EMT. These results are in agreement with the conclusion of a prior study showing that CTCs from patients with HER2 ${ }^{+}$ breast cancers were predominantly mesenchymal (22). CK expression in triple-negative tumor CTCs requires additional study and a larger sample population. Expression of PR indicates a functional ER $\alpha$ (one of the two isoforms of ER) and $\mathrm{ER} \alpha$ pathway (23), which increases E-cadherin expression by downregulating transcriptional repressors $(24,25)$. Patients with Her2-positive tumors lack ER and PR expression, which decreases the level of E-cadherin, and enhances the possibility of EMT and tumor cell invasion.

In patients with non-metastatic breast cancer, aneuploid CTCs independent of CK expression status can be detected by the iFISH system. Presence of CTCs and CTC counts was associated with tumor size and hormone receptor status in patients with breast cancer. Triploid CTCs constituted the majority of CTCs detected in all the patients with breast cancer evaluated regardless of hormone receptor status and tumor size. In addition, CK-positive CTCs were identified in a small cohort of patients and were detected at a low rate in CTC counts. Notably, CK expression was rare in CTCs from Her2-positive or triple-negative patients, supporting the hypothesis that lack of ER and PR may promote EMT and enhance tumor aggression.

\section{Acknowledgements}

The present study was funded by the National Natural Science Foundation of China (grant no. 81502546).

\section{References}

1. McGuire S: World Cancer Report 2014. Geneva, Switzerland: World Health Organization, International Agency for Research on Cancer, WHO Press, 2015. Adv Nutr 7: 418-419, 2016.

2. Xue P, Wu Y, Guo J and Kang Y: Highly efficient capture and harvest of circulating tumor cells on a microfluidic chip integrated with herringbone and micropost arrays. Biomed Microdevices 17: 39, 2015.

3. Mikolajczyk SD, Millar LS, Tsinberg P, Coutts SM, Zomorrodi M, Pham T, Bischoff FZ and Pircher TJ: Detection of EpCAM-negative and cytokeratin-negative circulating tumor cells in peripheral blood. J Oncol 2011: 252361, 2011.

4. Murray NP, Miranda R, Ruiz A and Droguett E: Diagnostic yield of primary circulating tumor cells in women suspected of breast cancer: The BEST (Breast Early Screening Test) study. Asian Pac J Cancer Prev 16: 1929-1934, 2015. 
5. Ge F, Zhang H, Wang DD, Li L and Lin PP: Enhanced detection and comprehensive in situ phenotypic characterization of circulating and disseminated heteroploid epithelial and glioma tumor cells. Oncotarget 6: 27049-27064, 2015

6. Harris L, Fritsche H, Mennel R, Norton L, Ravdin P, Taube S, Somerfield MR, Hayes DF and Bast RC Jr; American Society of Clinical Oncology: American Society of Clinical Oncology 2007 update of recommendations for the use of tumor markers in breast cancer. J Clin Oncol 25: 5287-5312, 2007.

7. Chen M, Palleschi S, Khoynezhad A, Gecelter G, Marini CP and Simms HH: Role of primary breast cancer characteristics in predicting positive sentinel lymph node biopsy results: A multivariate analysis. Arch Surg 137: 606-610, 2002.

8. Mego M, Giordano A, De Giorgi U, Masuda H, Hsu L, Giuliano M, Fouad TM, Dawood S, Ueno NT, Valero V, et al Circulating tumor cells in newly diagnosed inflammatory breast cancer. Breast Cancer Res 17: 2, 2015.

9. Yi M, Krishnamurthy S, Kuerer HM, Meric-Bernstam F, Bedrosian I, Ross MI, Ames FC, Lucci A, Hwang RF and Hunt KK: Role of primary tumor characteristics in predicting positive sentinel lymph nodes in patients with ductal carcinoma in situ or microinvasive breast cancer. Am J Surg 196: 81-87, 2008.

10. Alix-Panabières $\mathrm{C}$ and Pantel K: Circulating tumor cells: Liquid biopsy of cancer. Clin Chem 59: 110-118, 2013

11. Sheng Y, Wang T, Li H, Zhang Z, Chen J, He C, Li Y, Lv Y, Zhang J, Xu C, et al: Comparison of analytic performances of Cellsearch and iFISH approach in detecting circulating tumor cells. Oncotarget 8: 8801-8806, 2017.

12. Hillig T, Horn P, Nygaard AB, Haugaard AS, Nejlund S, Brandslund I and Sölétormos G: In vitro detection of circulating tumor cells compared by the CytoTrack and CellSearch methods. Tumour Biol 36: 4597-4601, 2015.

13. Min H, Jo SM and Kim HS: Efficient capture and simple quantification of circulating tumor cells using quantum dots and magnetic beads. Small 11: 2536-2542, 2015.

14. Wu C, Hao H, Li L, Zhou X, Guo Z, Zhang L, Zhang X, Zhong W, Guo H, Bremner RM and Lin P: Preliminary investigation of the clinical significance of detecting circulating tumor cells enriched from lung cancer patients. J Thorac Oncol 4: 30-36, 2009.
15. Li Y, Zhang X, Ge S, Gao J, Gong J, Lu M, Zhang Q, Cao Y, Wang DD, Lin PP and Shen L: Clinical significance of phenotyping and karyotyping of circulating tumor cells in patients with advanced gastric cancer. Oncotarget 5: 6594-6602, 2014.

16. Zhang Y, Wang F, Ning N, Chen Q, Yang Z, Guo Y, Xu D, Zhang D, Zhan T and Cui W: Patterns of circulating tumor cells identified by CEP8, CK and CD45 in pancreatic cancer. Int J Cancer 136: 1228-1233, 2015 .

17. Cao Y, Paner GP and Rajan PB: Sentinel node status and tumor characteristics: A study of 234 invasive breast carcinomas. Arch Pathol Lab Med 129: 82-84, 2005.

18. Allred DC, Clark GM, Tandon AK, Molina R, Tormey DC, Osborne CK, Gilchrist KW, Mansour EG, Abeloff M, Eudey L, et al: HER-2/neu in node-negative breast cancer: Prognostic significance of overexpression influenced by the presence of in situ carcinoma. J Clin Oncol 10: 599-605, 1992.

19. Sjögren S, Inganäs M, Lindgren A, Holmberg L and Bergh J: Prognostic and predictive value of c-erbB-2 overexpression in primary breast cancer, alone and in combination with other prognostic markers. J Clin Oncol 16: 462-469, 1998

20. Foulkes WD, Smith IE and Reis-Filho JS: Triple-negative breast cancer. N Engl J Med 363: 1938-1948, 2010.

21. Voduc KD, Cheang MC, Tyldesley S, Gelmon K, Nielsen TO and Kennecke $\mathrm{H}$ : Breast cancer subtypes and the risk of local and regional relapse. J Clin Oncol 28: 1684-1691, 2010

22. Yu M, Bardia A, Wittner BS, Stott SL, Smas ME, Ting DT, Isakoff SJ, Ciciliano JC, Wells MN, Shah AM, et al: Circulating breast tumor cells exhibit dynamic changes in epithelial and mesenchymal composition. Science 339: 580-584, 2013.

23. Cui X, Schiff R, Arpino G, Osborne CK and Lee AV: Biology of progesterone receptor loss in breast cancer and its implications for endocrine therapy. J Clin Oncol 23: 7721-7735, 2005.

24. Thomas C and Gustafsson JA: The different roles of ER subtypes in cancer biology and therapy. Nat Rev Cancer 11: 597-608, 2011.

25. Ye Y, Xiao Y, Wang W, Yearsley K, Gao JX, Shetuni B and Barsky SH: ERalpha signaling through slug regulates E-cadherin and EMT. Oncogene 29: 1451-1462, 2010. 